\title{
NOTES AND CORRESPONDENCE
}

\section{Influence of the Aleutian-Icelandic Low Seesaw and ENSO onto the Stratosphere in Ensemble Winter Hindcasts}

\author{
Yvan J. ORSOLINI \\ Norwegian Institute for Air Research, Kjeller, Norway \\ Nils Gunnar KVAMSTØ \\ Geophysical Institute, University of Bergen, Bergen, Norway \\ Ina T. KINDEM \\ Bjerknes Centre for Climate Research, Bergen, Norway \\ Meiji HONDA \\ JAMSTEC, Frontier Research Center for Global Change, Yokohama, Japan \\ and \\ Hisashi NAKAMURA \\ Department of Earth and Planetary Sciences, The University of Tokyo, Tokuo, Japan \\ JAMSTEC, Frontier Research Center for Global Change, Yokohama, Japan
}

(Manuscript received 16 August 2007, in final form 7 June 2008)

\begin{abstract}
Using an ensemble of wintertime hindcasts with a high-resolution (T106L60) Atmospheric General Circulation Model (AGCM) forced by observed sea surface temperatures (SSTs) and extending into the stratosphere, we investigate the formation and lifecycle of the Aleutian-Icelandic low Seesaw (AIS) during the 1978 to 1993 period. The AIS has been newly proposed to be an important mode of variability, linking the major wintertime surface lows, the Icelandic Low and the Aleutian Low, in late winter, and thereby linking climate variability over the North Pacific and the North Atlantic. We demonstrate for the first time with a stratosphere-troposphere model, that a coherent, ensemble-mean AIS extension into the stratosphere exists, where its presence modulates ultra-long planetary wave propagation and the polar night jet intensity. The model AIS peaks in February, when the Aleutian and Icelandic Low anti-correlation maximizes at -0.59 . The AIS provides a new way to describe the El Niño-Southern Oscillation (ENSO) phenomenon influence into the stratosphere. For example, El-Nino conditions correspond to a deeper than normal Aleutian Low, extending its influence into the Icelandic sector as an AIS negative phase (weakened Icelandic Low), hence enhanced planetary wave vertical propagation and a weakened stratospheric polar vortex. This maturation of the AIS in late winter explains the intra-seasonal variability of the stratospheric response to ENSO, which peaks in late winter. Internal model variability is large and enhanced potential predictability is found primarily in the western hemisphere, with a western Atlantic maxima being more pronounced in the stratosphere than in the upper troposphere.
\end{abstract}




\section{Introduction}

Observational and model evidences indicate that inter-annual climate variations over the North Pacific and Atlantic sectors are coupled in late winter (e.g., Van Loon and Rogers 1978). Recently, Honda et al. (2001), Honda and Nakamura (2001) and Nakamura and Honda (2002) used meteorological reanalyses from the U.S. National Centers for Environmental Prediction (NCEP) and the U.S. National Center for Atmospheric Research (NCAR) to show that, in February and March, an interannual seesaw exists between the strengths of the two major climatological surface low-pressure cells in the northern hemisphere, namely the Aleutian Low (AL) and the Icelandic Low (IL). They named this seesaw the AIS. Using the ERA-40 reanalyses from the European Centre for Medium-Range Weather Forecasts (ECWMF), Orsolini (2004) further demonstrated that the AL-IL late-winter anticorrelation persists for the period 1959-2002 (-0.39 in February), albeit it is weaker than for the 1973 -1994 period used by Honda et al. (2001) (-0.67 in February).

The AIS appears through the time development of early winter Pacific anomalies and their subsequent extension into the Euro-Atlantic sector as a Pacific-North American (PNA) pattern. Excited by the El Niño/Southern Oscillation (ENSO), the PNA is a spatial pattern that emerges among the leading northern hemispheric modes of variability in empirical orthogonal function analyses or in onepoint regression maps (Wallace and Gutzler 1981; Ambaum et al. 2001).

The AIS involves the horizontal propagation of planetary-scale waves, and their interaction with migrating, synoptic-scale eddies over both ocean basins. The AIS also influences the propagation of ultra-long planetary waves into the stratosphere and the intensity of the polar night jet (Nakamura and Honda 2002). The seesaw is hence of great importance in understanding the stratospheretroposphere coupling and large-scale anomalies in column ozone (Orsolini 2004).

In addition to investigating the AIS in multidecadal reanalyses, Honda et al. (2005a) examined

Corresponding author: Yvan J. Orsolini, Norwegian Institute for Air Research, Instituttveien 18, P.O.BOX. 100, N-2027, Kjeller, Norway

E-mail: orsolini@nilu.no

(C)2008, Meteorological Society of Japan the seesaw formation and its predictability in ensemble AGCM simulations using prescribed sea surface temperatures (SSTs) and sea-ice conditions. Their 24-member ensemble of 50-year simulations revealed North Pacific-North Atlantic coupling in the form of a coherent AIS signal, excited by the lower boundary forcings. In their model, low potential predictability was found over the North Atlantic region in the upper troposphere, due to a large variability among ensemble members.

The influence of ENSO on the modelled or observed stratospheric circulation has also received renewed attention since van Loon and Labitzke (1987) showed that El-Niño events were associated with a weakened polar vortex. While early studies on the ENSO-stratosphere connection had difficulties to establish statistical significance using short time span of re-analyses (e.g., see Hamilton 1993; Baldwin and O'Sullivan 1995), more recently, Camp and Tung (2007) used 47 years of NCEP temperature data to relate El-Niño events to stratospheric warm anomalies. They further showed the magnitude of this influence to be comparable to the one exerted by the Quasi-Biennal Oscillation (QBO). Using two different GCMs extending into the middle atmosphere, Sassi et al. (2004) and Manzini et al. (2006) demonstrated that the El-Niño phase of ENSO is associated with enhanced vertical planetary wave propagation, a weaker than normal stratospheric polar night jet (PNJ) and a warmer polar stratosphere. Garcia-Herrera et al. (2006) used the Whole-Atmosphere-Community-Climate Model (NCAR WACCM), the Max-Planck-Institute for Meteorology ECHAM5 model, and the ERA-40 reanalyses to emphasize that the ENSO temperature signal propagated more efficiently into the stratosphere from the mid latitudes in winter, when zonal-mean winds favour upward propagation. In a case study of the exceptional El-Niño event of 1940 -42 , Bronnimann et al. (2004) also showed that an anomalously deep Aleutian Low and an anomalously weak Icelandic Low coexisted with a weakened stratospheric polar vortex. Using the NCEP/NCAR and ERA-40 re-analyses, Limpasuvan et al. (2005) showed that an anomalously strong $50-\mathrm{mb}$ PNJ is more common in La-Niña phase. In the model study of Manzini et al. (2006), by contrast, the response to ENSO was asymmetric, with no clear signal in the La-Niña phase.

The AIS connection to the ENSO phenomenon and to the winter stratospheric variability warrants a study using a model that extends into the strato- 
sphere. To this end, we have investigated the AIS in a series of winter ensemble hindcasts with a high-resolution AGCM externally forced with observed SST and sea ice. Our model differs notably from the simulations used in Honda et al. (2005a), in that we carried out seasonal, winter (DJFM) runs for a 14-year period, at a much higher horizontal resolution and with a well-resolved stratosphere. No other model study has investigated the AIS signature in the stratosphere.

\section{Model runs}

Our ensemble of winter simulations with the “Arpege Climat" AGCM (V3.0), with 60 vertical levels from the surface to $0.2 \mathrm{mb}$, at T106 horizontal resolution ( $1.5 \times 1.5$ degrees) covers the 14 winter seasons of the European Re-Analyses ERA-15 period, from $1979 / 80$ to $1992 / 93$. Background information on the model can be found in Deque et al. (1994) or Cassou and Terray (2001). An ensemble of nine members was generated by time-shifting initial conditions. For comparison, the 24-member 50-year run in Honda et al. (2005a) had 19 levels and a T42 horizontal resolution, while the 3member 51-year run in Sassi et al. (2004) had 66 levels up to $140 \mathrm{~km}$, and a T63 horizontal resolution. Observed monthly-mean SSTs and sea-ice from the Reynolds dataset were updated daily in the model through linear interpolation. Stratospheric initial conditions were taken from the ERA-40 data. Ozone is prescribed to climatological values.

While monthly-mean, ensemble-mean fields are mostly used in this study, we also carried out an analysis of variance using individual monthly means from all members.

\section{The AIS and its life cycle}

To derive a model AIS index, we first locate the winter climatological SLP centres of the AL and IL. The AL and IL indices are defined as SLP averages over the regions of maximum variance in these sectors. In the North Atlantic, the maximum variance is between Iceland and Greenland, on the northwestern flank of the IL climatological centre. The IL index is hence defined as SLP averaged over $315 \mathrm{E}-345 \mathrm{E}$ and $55 \mathrm{~N}-65 \mathrm{~N}$. In the Pacific, maximum variance is found south of the Aleutian Islands, and the AL index is defined as SLP averaged over $185 \mathrm{E}$ $-215 \mathrm{E}$ and $50 \mathrm{~N}-60 \mathrm{~N}$. The AIS index is the difference between the normalised AL and IL indices (i.e., AL-IL). A positive value of the AIS index thus corresponds to a weaker AL and an intensified IL. Nakamura and Honda (2002) showed that the AIS is a robust feature, and does not strongly depend on being calculated using averaged or minimum SLP values in a domain. To diagnose the externally forced component of the seesaw, we examined the ensemble-mean IL and AL standardized fluctuations in each winter month, over the 14 years. Yearto-year variations in the AL and IL show a clear anti-correlation, with an intra-seasonal modulation: -0.48 (December), -0.43 (January), -0.59 (February), and -0.37 (March). The seesaw peak period is also February in observations (Honda et al., 2001, 2005b). Figure 1 shows the AL, IL and AIS
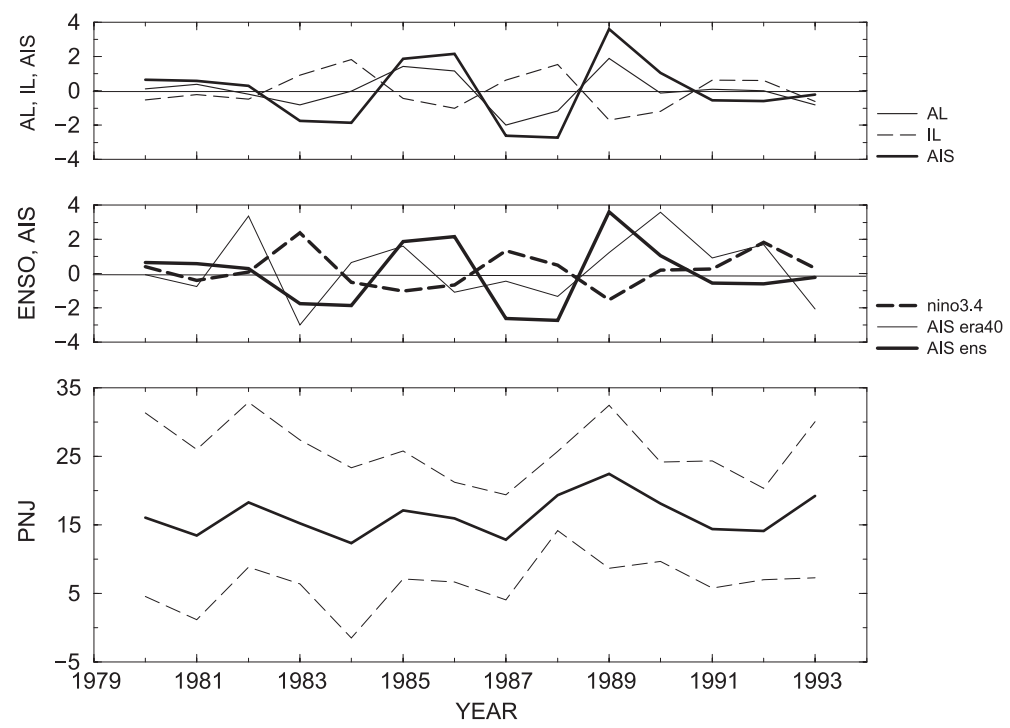

Fig. 1. (TOP) Ensemble-mean Aleutian (AL) and Icelandic (IL) SLP normalised anomalies in February, and the AIS index, AL minus IL. (MIDDLE) February AIS indices for the model (as above) and ERA-40, and winter-mean (DJF) El Niño 3.4 index. (BOTTOM) February PNJ (i.e., zonal-mean zonal wind at $50 \mathrm{mb}$ and $60 \mathrm{~N}$, in $\mathrm{ms}^{-1}$ ) in the model ensemble-mean (thick black line). The dashed lines give the PNJ range among the ensemble for each year, as an indicator of internal variability. 
model time series in February (TOP). On Fig. 1 (MIDDLE), the model AIS index (thick line) is shown again with the ERA-40 AIS index (thin line). The later shows little correspondence with the model one. For example, the two years when the AIS index is the largest are 1982 and 1990 in ERA40, but 1986 and 1989 in the model. The difficulty in hindcasting the IL and AL intensities despite the forced SSTs, was also noted by Honda et al. (2005a). Seasonal hindcasts of the NAO with prescribed SSTs have also shown only moderate skill (e.g., Doblas-Reyes et al. 2003). In this paper, we are interested in the AIS as a model mode of variability rather than in actual AIS seasonal forecast.

The model ensemble-mean geopotential heights at $200 \mathrm{mb}$ show a coherent seesaw life cycle that develops into the late-winter AL and IL anticorrelation. The composite differences in Fig. 2, aggregated when the SLP-based AIS index is large in February (above 0.67 or below -0.67), and then lagged to reveal the evolution of anomalies from December to March, show a seasonal evolution similar to the ensemble-mean life cycle in the modelling study of Honda et al. (2005a). It is also in good agreement with the observational coun-
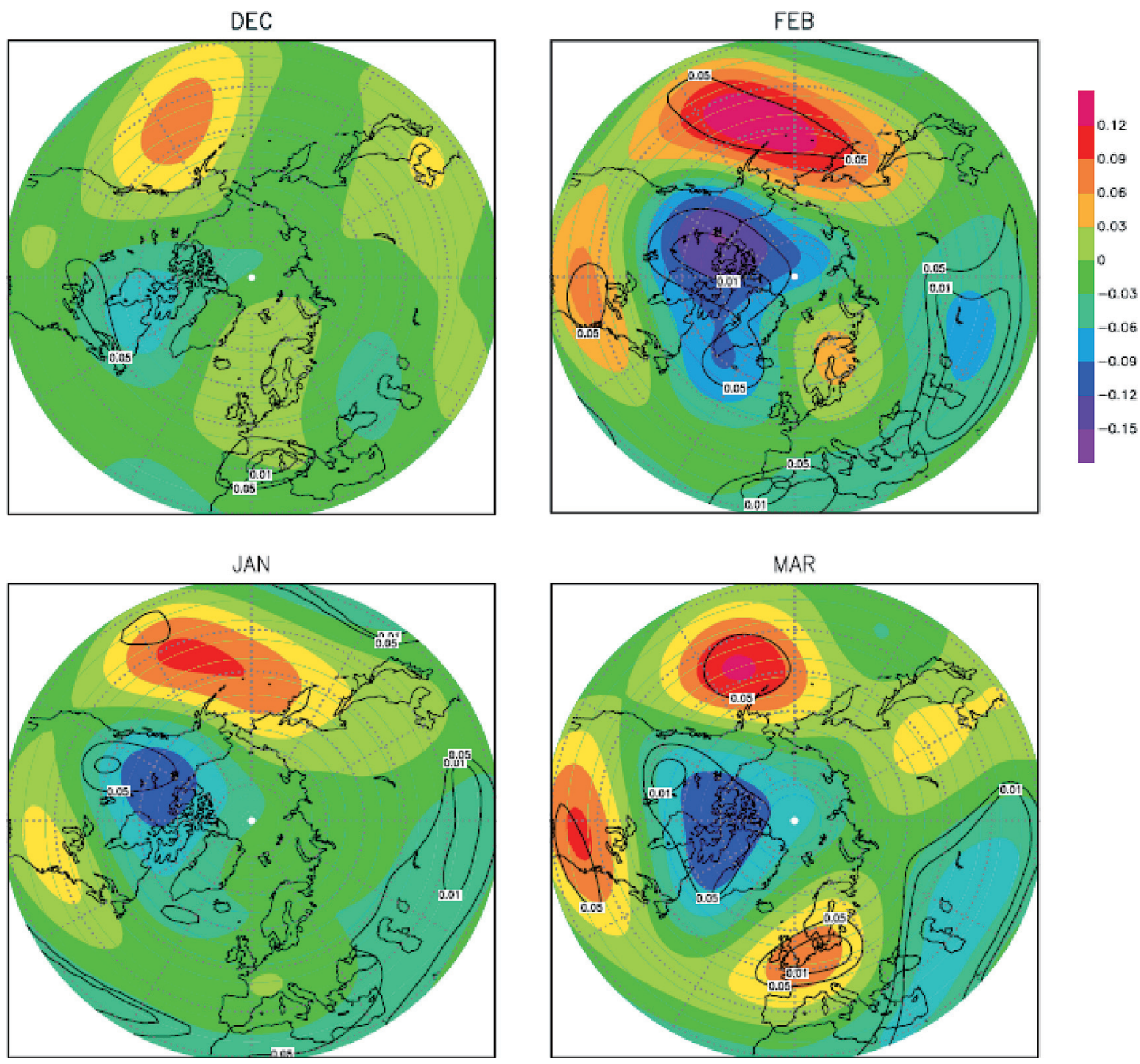

Fig. 2. Ensemble-mean composite differences in geopotential height $(200 \mathrm{mb})$ showing the externally forced life-cycle of the seesaw from December through March, based on February values of the AIS index. Units are $\mathrm{km}$. Color scale is from -0.15 to 0.12 . Black contours indicate statistical significance at the $95 \%$ and $99 \%$ level based on t-tests. 
terpart shown by Honda et al. (2001, 2005b), albeit both model studies show weaker amplitudes over the Atlantic. Precursory positive anomalies appear over the Pacific in December, intensify in January, giving rise to a nascent PNA-like wave train in December and in January. A secondary wave train emanates in February, extending across the North Atlantic and Europe, at the peak of the seesaw. It is still prominent in March. Anomalies are statistically significant during the peak of the seesaw.

The horizontal propagation of anomalous wave trains emanating from early winter anomalies over the Pacific toward the Euro-Atlantic sector in the ensemble mean is supported by upper-troposphere wave activity flux calculations. To this end, the methodology of Takaya and Nakamura (2001) is used, which allows wave fluxes to be associated with departures from a non-zonal, time-mean basic state. Figure 3 shows the monthly evolution of both the horizontal (at $200 \mathrm{mb}$ ) and the vertical (at 100 $\mathrm{mb}$ ) components of the wave flux, throughout the ensemble-mean AIS lifecycle. Local geostrophic balance has been assumed to derive horizontal velocities. Downstream emanation of the flux from the North Pacific and ultimately its dissipation over the North Atlantic both strengthen during the AIS

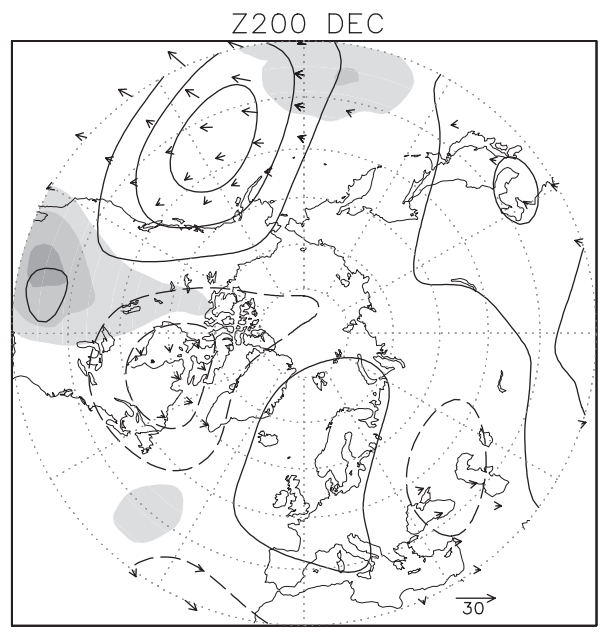

$\begin{array}{llllll}10 & 20 & 30 & 40 & 50 & 60\end{array}$

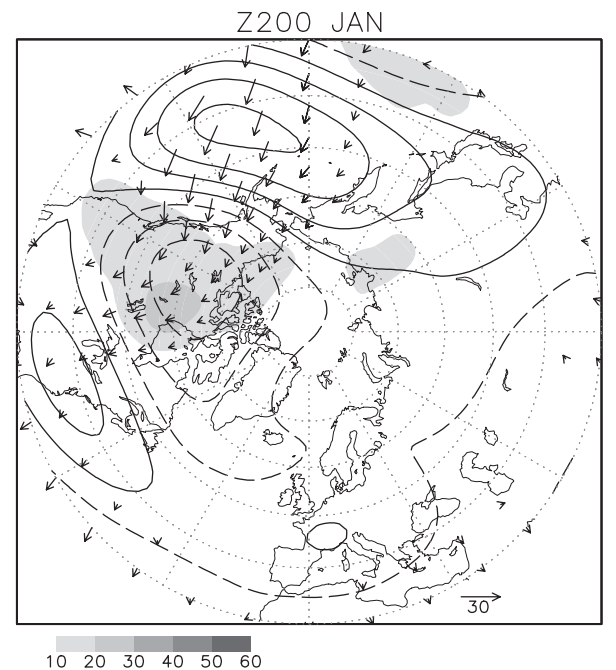

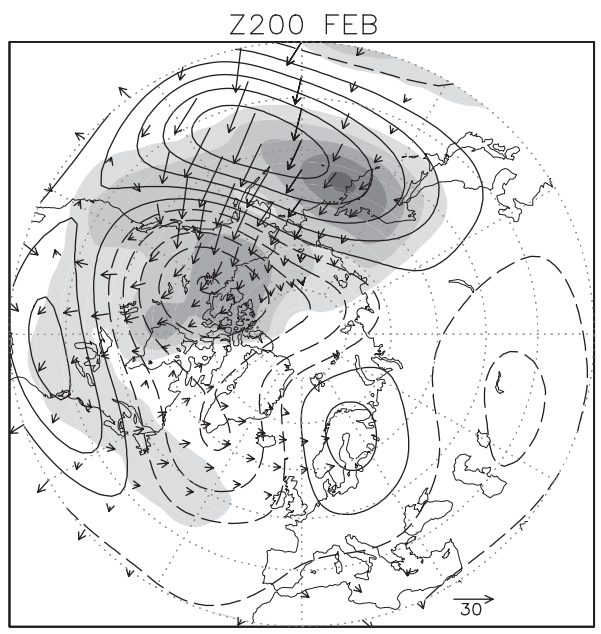

$\begin{array}{llllll}10 & 20 & 30 & 40 & 50 & 60\end{array}$

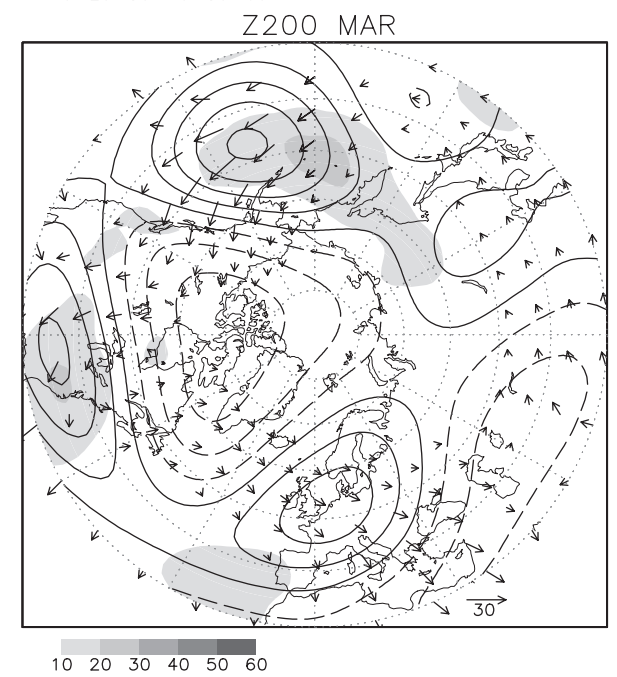

Fig. 3. Horizontal component of the Takaya-Nakamura wave activity flux at $200 \mathrm{mb}$ for the ensemble-mean monthly means, from December through March (units of $\mathrm{m}^{2} \mathrm{~s}^{-2}$, arrow scale indicated). Ensemblemean 200mb-geopotentail height anomalies are also indicated (thin lines, contours are $0.03 \mathrm{~km}$ ). Vertical component of the wave activity flux at $100 \mathrm{mb}$ is grey shaded (units are $10^{-3} \mathrm{Nm}^{3} \mathrm{~s}^{-2}$ ) 
lifecycle. Vertical propagation maximizes during the mature phases of the seesaw, over the North Pacific and Northwestern America.

The stratospheric signal of the seesaw is evident in the composite difference of the 30-mb geopotential height anomalies in Fig. 4. In the positive phase of the seesaw lifecycle, in February, polar cyclonic anomalies appear as vertical extensions of the 200-mb cyclonic anomalies seen in Fig. 2. They maximize in the high latitudes of North America. In the negative phase, the anticyclonic anomalies in the stratosphere are associated with a weakened polar vortex displaced toward Eurasia, consistent with enhanced, deeper than normal AL and a stronger planetary wave- 1 . The amplitude of the anomalies, on the order of $300 \mathrm{~m}$ at $30 \mathrm{mb}$, is consistent with those based on NCEP re-analyses in Nakamura and Honda (2002), albeit the latter show a stronger wave-2 component, perhaps due to vortex variability during the more extended period (19671997). Upward flux at $100 \mathrm{mb}$, shown again on Fig. 4 , corresponds to the divergence of the horizontal flux at $30 \mathrm{mb}$. The AIS is associated with polar temperature anomalies at $30 \mathrm{mb}$ (Fig. 5). The peak $4 \mathrm{~K}$ cooling (warming) in the AIS positive (negative)

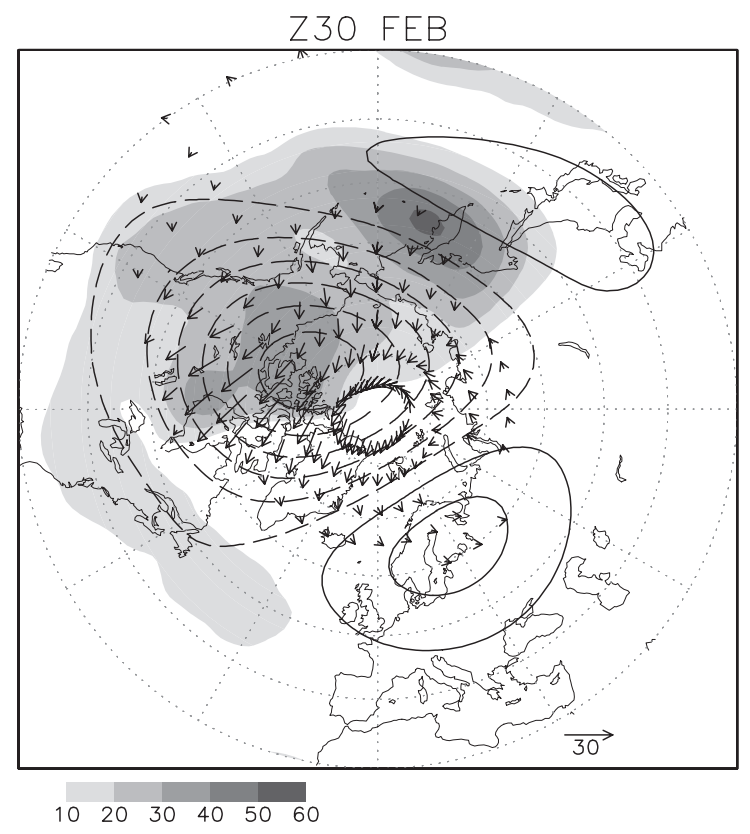

Fig. 4. Same as Fig. 2 for $30 \mathrm{mb}$ geopotential height, in February (units are $\mathrm{km}$ ). Vertical wave activity flux at $100 \mathrm{mb}$ is also indicated (as in Fig. 3). phase over the North Pacific is slightly larger than in the observational study of Nakamura and Honda (2002), but comparable to the model study of Manzini et al. (2006). Opposite temperature anomalies are found over the North Atlantic.

\section{Connection to ENSO}

As seen in Fig. 1 (MIDDLE), the ensemble-mean AIS in February is highly anti-correlated $(-0.69)$ with the ENSO phenomenon. Here, we represent ENSO by the winter-mean (DJF) El-Niño 3.4 index, an average of SSTs over the $5 \mathrm{~N}-5 \mathrm{~S}, 120 \mathrm{~W}-170 \mathrm{~W}$ tropical Pacific area. Cold SSTs (La Niña) are linked to a weaker than normal AL, corresponding to the positive phase of AIS. We further show in Fig. 1 (BOTTOM) that La Niña (El Niño) years with the positive (negative) AIS phase are associated with a stronger (weaker) than normal PNJ, associated with the cyclonic (anticyclonic) anomalies in the stratosphere over the Arctic (Fig. 4). The correlation between the ensemble-mean $50-\mathrm{mb}$ zonal-mean zonal wind at $60 \mathrm{~N}$, as a measure of the PNJ intensity, and the ENSO index is -0.39 in February. This PNJ index also correlates with the AIS index ( 0.5 in February), with its positive phase and weaker AL, associated with lessened vertical planetary wave propagation and a stronger PNJ (Nakamura and Honda 2002; Orsolini 2004). The correlation between the ERA-40 (1958-2002) PNJ intensity in February and the El-Niño index is -0.32 , close to our model ensemble-mean results

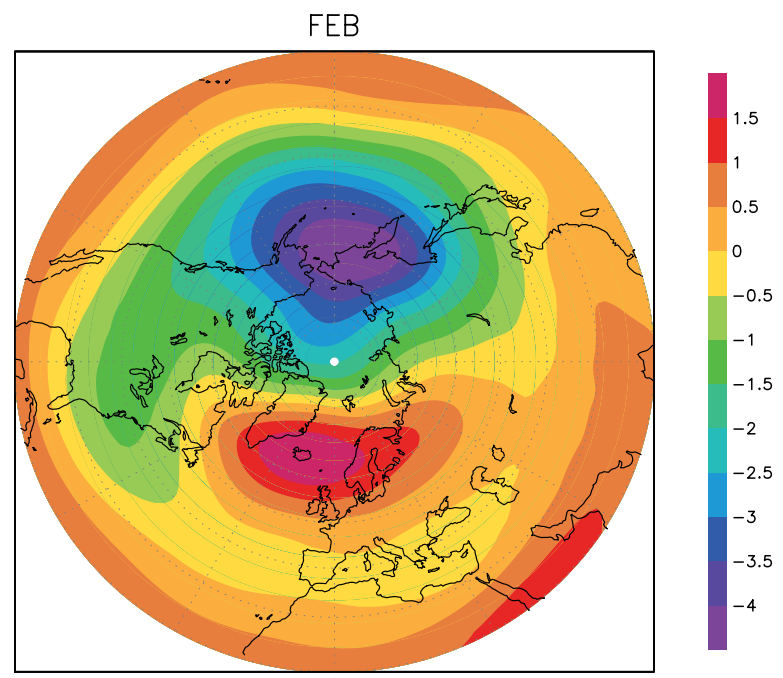

Fig. 5. Same as Fig. 3 for temperature at $30 \mathrm{mb}$ (units are $\mathrm{K}$ ). 
$(-0.39)$. In addition, the sign of this correlation between the PNJ intensity and ENSO is the same as in the more recent observational and model studies mentioned in Section 1: e.g., a stronger polar vortex during La-Niña events, versus an anomalous high over northern Canada and the displaced vortex during El-Niño events. There is, in particular, quantitative agreement between our mid-stratospheric temperature AIS composites and the ENSO composites in Manzini et al. (2006). Difference of upper-tropospheric geopotential height anomalies in AIS composites in Fig. 2 are also consistent with the PNA-like pattern in Taguchi and Hartmann's (2006) ENSO composites from their idealised, perpetual January model simulations. The consideration of the AIS life-cycle development in the troposphere also helps explaining the seasonality of the ENSO strastospheric response, which is largest in February (Van Loon and Labitzke 1987).

\section{Potential predictability}

Potential predictability is often used in assessing how ensemble simulations are influenced by boundary forcings, such as SSTs, in comparison with the model internal variability, hence to separate the external variability from the internal variability. The former is the ensemble-mean year-toyear variability, while the latter characterises the departure of members from their ensemble mean arising from the model's non-linear dynamics. Potential predictability is the ratio of external to total (external plus internal) variance.

We first note that for the AIS index itself, internal and external variability are nearly equal, so that its potential predictability is around 0.5 ; it maximises in February (0.54). This is substantially higher than the potential predictability of the PNJ, which is only 0.13 . Hindcast maps of potential predictability for geopotential height were calculated for February both in the upper troposphere (200 $\mathrm{mb})$ and the stratosphere (30 mb) (Fig. 6). Each month, 126 departures of the height fields from their ensemble-mean (9 members times 14 years) were used for calculating the internal variance, while the 14 departures of the ensemble-mean height fields from their 14-year time average were used for the external variance. In the troposphere, higher values are found over the Pacific, extending from the subtropics toward N. America and the western Atlantic, along the wave train associated with the PNA pattern. Little of this predictability enhancement penetrates over the European sector
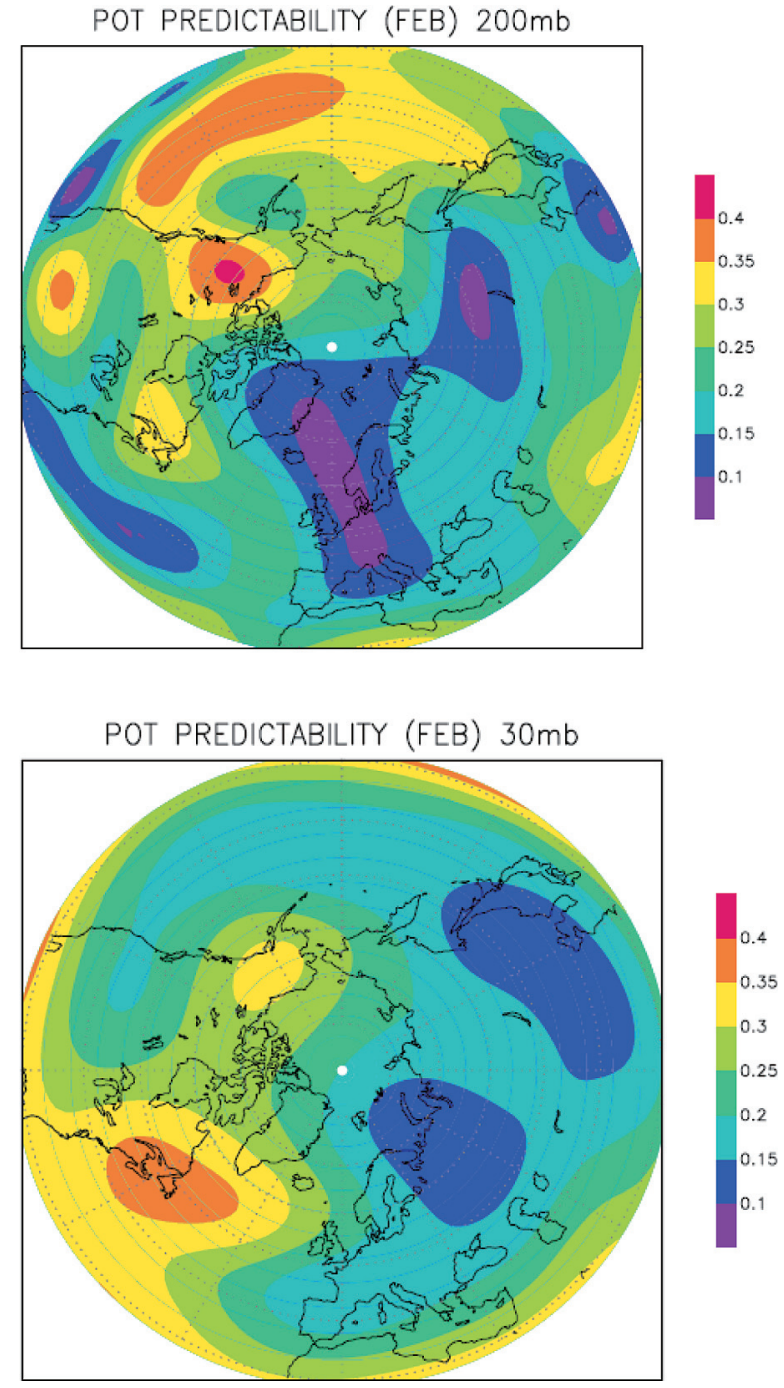

Fig. 6. Hindcast potential predictability maps (ratio of external to total variance) at $200 \mathrm{mb}$ and $30 \mathrm{mb}$ in February.

at $200 \mathrm{mb}$. At $30 \mathrm{mb}$, the potential predictability is higher over the western hemisphere, over the mid latitudes above the western Atlantic, and it extends further east toward Iceland than at $200 \mathrm{mb}$. These potential predictability maps indicate that the SSTs influence on the month-to-month stratospheric variability can be zonally confined, and could be better predicted in the western Euro-Atlantic sector. In that region, both observed and internal model stratospheric variability is reduced. 


\section{Conclusions}

The present analysis of ensemble-mean AGCM winter ensemble hindcasts over the years 1979 to 1993 (at high horizontal resolution and with a wellresolved stratosphere) shows:

i) a clear signature and coherent seasonal evolution of the AIS in the upper troposphere, involving the development of a PNA-like anomaly pattern, and its vertical propagation into the stratosphere

ii) a significant negative correlation between the AIS index and the El-Niño 3.4 index, with the warm phase of ENSO (El Niño) characterized by a deeper than normal AL, weaker IL and a weakened polar vortex. This maturation of the AIS in late winter could explain the intra-seasonal variability of the responses to ENSO, in the stratosphere and over the Atlantic sector in the troposphere.

While a coupled ocean-atmosphere model might be needed to properly represent ENSO dynamics, we nevertheless see in our GCM forced by observed SSTs that tropical Pacific SST anomalies associated with ENSO, set up a PNA-like pattern and an anomalous surface AL. During the AIS formation, planetary wave horizontal propagation in mid latitudes allows downstream influences from the North Pacific to the Icelandic sector, while the vertical propagation of ultra-long planetary waves modulates the PNJ intensity (e.g., more wave-1 during the AIS negative phase). Overall, a high degree of internal variability exists both in the stratosphere and troposphere, indicated by the low potential predictability. In limited observational time series, the small but coherent influence upon the PNJ arising from the tropical Pacific SSTs could be masked by the atmospheric non-linear internal dynamics and by other larger factors that affects the stratospheric vortex such as the QBO or the solar cycle.

In addition, the observed AIS signature displays some decadal modulations (Honda et al. 2005b) with an active phase in the last three decades, while being nearly absent in the previous two decades. The ERA-15 period for our hindcast study coincides with the active phase of the AIS, and future studies should further address the issue of modelled versus observed decadal modulations of AIS.

The linkage between the AIS and the PNJ fluc- tuations in response to ENSO has not been shown previously in stratospheric models. It is clearly seen in the model ensemble-mean, where the large model internal variability is removed, than in the real atmosphere, which is just one possible realisation. Our analysis indicates that the ensemblemean stratospheric circulation has a strong nonzonal response to the lower boundary forcing (SSTs), esp. in the western hemisphere, but also a weak response in the zonally-averaged PNJ.

Much of the recent research on stratospheretroposphere coupling has adopted the viewpoint of annular modes (e.g., Limpasuvan et al. 2005, and references therein). The AIS rather represents opposing year-to-year fluctuations between the North Pacific and Atlantic sectors in late winter, and hence emphasizes zonal wave-like teleconnections (out-of-phase) between the two ocean basins. On the contrary, the annular mode viewpoint rather highlights winter-mean meridional linkages between high and mid latitudes. In that respect, we note that, while the ensemble-mean PNJ shows a weak, passive response to El Niño forced by enhanced ultra-long planetary waves, it does not preclude that the stratosphere could exert a downward, re-inforcing influence onto the troposphere.

\section{Acknowledgements}

This work was supported by the Norwegian Research Council (project NAO) to NILU and the University of Bergen. We also acknowledge the Japan Society for Promotion of Science for supporting the lead author through a short-term fellowship at the University of Tokyo.

\section{References}

Ambaum, M., B.J. Hoskins, and D.B. Stephenson, 2001: Arctic Oscillation or North Atlantic Oscillation? J. Climate, 14, 3495-3507.

Baldwin, M.P. and D. O'Sullivan, 1995: Stratospheric effects of ENSO-related tropospheric circulation anomalies. J. Climate, 8, 649-667.

Bronnimann, S., J. Luterbacher, J. Staehelin, T.M. Svendby, G. Hansen, and T. Svenoe, 2004: Extreme climate of the global troposphere and stratosphere in 1940-42 related to El Niño. Nature, 431, 971-974.

Camp, C.D. and K.-K. Tung, 2007: Polar warming by ENSO in winter: a statistical study. Geophys. Res. Lett., 34, doi:10.1029/2006GL028521.

Cassou, C. and L. Terray, 2001: Oceanic forcing of the wintertime low-frequency atmospheric variability in the North Atlantic European Sector: a study 
with the Arpege model. J. Climate, 14, 42664291.

Deque, M., C. Dreveton, A. Braun, and D. Cariolle, 1994: The climate version of the Arpege/IFS: a contribution to the French community climate modelling. Clim. Dyn., 10, 249-266.

Doblas-Reyes, F.J., V. Pavan, and D.B. Stephenson, 2003: The skill of multi-model seasonal forecasts of the wintertime North Atlantic Oscillation. Clim. Dyn., 21, 501-514.

García-Herrera, R., N. Calvo, R. R. Garcia, and M. A. Giorgetta, 2006: Propagation of ENSO temperature signals into the middle atmosphere: A comparison of two general circulation models and ERA-40 reanalysis data. J. Geophys. Res., 111, D06101, doi:10.1029/2005JD006061.

Hamilton, K., 1993: An examination of the observed Southern Oscillation effects in the northern hemisphere stratosphere. J. Atmos. Sci., 50, 3468 -3473 .

Honda, M., H. Nakamura, J. Ukita, I. Kousaka, and K. Takeuchi, 2001: Interannual seesaw between the Aleutian and Icelandic Lows. Part I : Seasonal dependence and life cycle. J. Climate, 14, 10291042.

Honda, M. and H. Nakamura, 2001: Interannual seesaw between the Aleutian and Icelandic Lows. Part II: Its significance in the interannual variability over the wintertime northern hemisphere. J. Climate, 14, 4512-4529.

Honda, M., Y. Kushnir, H. Nakamura, S. Yamane, and S.E. Zebiak, 2005a: Formation, mechanisms and predictability of the Aleutian-Icelandic Low seesaw in ensemble AGCM simulations. J. Climate, 18, 1423-1434.

Honda, M., S. Yamane, and H. Nakamura, 2005b: Impacts of the Aleutian-Icelandic Low seesaw on surface climate during the twentieth century. $J$.
Climate, 18, 2793-2802.

Limpasuvan, V., D.L. Hartmann, D.W.J. Thompson, K. Jeev, and Y.L. Yung, 2005: Stratosphere-troposphere evolution during polar vortex intensification. J. Geophys. Res., 110, D24101, doi:10.1029/ 2005JD006302.

Manzini, E., M.A. Giorgetta, M. Esch, L. Kornblueh, and E. Roeckner, 2006: The influence of sea surface temperatures on the northern winter stratosphere: ensemble simulations with the MAECHAM5 model. J. Climate, 19, 3863-3881.

Nakamura, H. and M. Honda, 2002: Interannual seesaw between the Aleutian and Icelandic Lows. Part III : Its influence upon the stratospheric variability. J. Meteor. Soc. Japan, 80, 1051-1067.

Orsolini, Y.J., 2004: Seesaw Ozone Fluctuations between the North Pacific and Atlantic in Late Winter. J. Meteor. Soc. Japan, 82, 941-948.

Sassi, F., D. Kinnison, B.A. Boville, R.R. Garcia, and R. Roble, 2004: Effects of El Niño-Southern Oscillation in the dynamical, thermal, and chemical structure of the middle atmosphere. J. Geophys. Res., 109, D17108, doi:10.1029/2003JD004434.

Takaya, T. and H. Nakamura, 2001, A formulation of a phase-independent wave-activity flux for stationary and migratory quasigeostrophic eddies on a zonally varying basic flow. J. Atmos. Sci., 58, 608627.

van Loon, H. and K. Labitzke, 1987: The Southern Oscillation, part V: The anomalies in the lower stratosphere of the Northern Hemisphere in winter, and a comparison with the Quasi-Biennal Oscillation. Mon. Wea. Rev., 115, 357-369.

Wallace, J.M. and D.S. Gutzler, 1981: Teleconnections in the geopotential height field during the northern hemisphere winter. Mon. Wea. Rev., 109, 784 -812 . 